\title{
First isomeric quadrupole moment measured in fragmentation reactions: The case of ${ }^{61} \mathrm{Fe}^{m}\left(9 / 2^{+}\right)$
}

\author{
N. Vermeulen, ${ }^{1}$ S. K. Chamoli, ${ }^{2}$ J. M. Daugas, ${ }^{3}$ M. Hass, ${ }^{2}$ D. L. Balabanski, ${ }^{4,5}$ J. P. Delaroche, ${ }^{3}$ F. de Oliveira-Santos, ${ }^{6}$ \\ G. Georgiev, ${ }^{7}$ M. Girod, ${ }^{3}$ G. Goldring, ${ }^{2}$ H. Goutte,${ }^{3}$ S. Grévy, ${ }^{6}$ I. Matea, ${ }^{8}$ P. Morel, ${ }^{3}$ B. S. Nara Singh, ${ }^{2}$ Yu.-E. Penionzkevich, ${ }^{9}$ \\ L. Perrot, ${ }^{6}$ O. Perru, ${ }^{3}$ S. Péru, ${ }^{3}$ O. Roig, ${ }^{3}$ F. Sarazin, ${ }^{10}$ G. S. Simpson, ${ }^{11}$ Yu. Sobolev, ${ }^{9}$ I. Stefan, ${ }^{6}$ C. Stodel,${ }^{6}$ \\ D. T. Yordanov, ${ }^{1}$ and G. Neyens ${ }^{1}$ \\ ${ }^{1}$ Instituut voor Kern-en Stralingsfysica, K. U. Leuven, B-3001 Leuven, Belgium \\ ${ }^{2}$ Department of Particle Physics, Weizmann Institute of Science, Rehovot 76100, Israel \\ ${ }^{3}$ CEA/DIF/DPTA/PN, BP 12, F-91680 Bruyères le Châtel, France \\ ${ }^{4}$ Dipartimento di Fisica, Universitá di Camerino, I-62032 Camerino, Italy \\ ${ }^{5}$ Institute for Nuclear Research and Nuclear Energy, Bulgarian Academy of Sciences, 1784 Sofia, Bulgaria \\ ${ }^{6}$ GANIL, BP 55027, F-14076 Caen Cedex 5, France \\ ${ }^{7}$ ISOLDE, CERN, 1211 Geneva 23, Switzerland \\ ${ }^{8}$ CENBG, Le Haut Vigneau, BP 120, F-33175 Gradignan Cedex, France \\ ${ }^{9}$ FLNR-JINR, RU-141980 Dubna, Russia \\ ${ }^{10}$ Colorado School of Mines, Department of Physics, Golden, Colorado 80401, USA \\ ${ }^{11}$ Institut Laue-Langevin, BP 156, F-38042 Grenoble Cedex 9, France \\ (Received 27 October 2006; published 14 May 2007)
}

\begin{abstract}
This paper demonstrates the feasibility of quadrupole moment measurements on isomeric levels populated in projectile fragmentation reactions. The neutron-rich ${ }^{61} \mathrm{Fe}\left(9 / 2^{+}\right)\left[E^{*}=861 \mathrm{keV}, T_{1 / 2}=239(5) \mathrm{ns}\right]$ isomer was produced and spin aligned by the intermediate energy fragmentation of a ${ }^{64} \mathrm{Ni}$ beam and implanted in a $\mathrm{Cd}$ single crystal. Its spectroscopic quadrupole moment $\left|Q_{s}\right|=41(6) e \mathrm{fm}^{2}$ agrees with mean-field based calculations using the finite-range Gogny force, suggesting a moderately deformed shape characterized by an intrinsic charge quadrupole moment $Q_{0}=-85 e \mathrm{fm}^{2}$ or $Q_{0}=+115 e \mathrm{fm}^{2}$. The present measurement paves the way for future determinations of isomeric quadrupole moments in more exotic nuclei.
\end{abstract}

DOI: 10.1103/PhysRevC.75.051302

PACS number(s): 21.10.Ky, 21.60.-n, 23.20.En, 27.50.+e

One of the most fundamental characteristics of a nucleus is its shape. Nuclear properties, ranging from electromagnetic moments and transition probabilities to half-lives and energy levels, display a strong dependence on the nuclear deformation. Various collective models, based on assuming a specific nuclear shape, have been successful in explaining a wide variety of nuclear features. These models, however, do not explain the origin of the deformation. The task of unraveling the microscopic mechanisms that cause deformation forms a large part of modern nuclear structure research, both theoretically and experimentally. The nuclear quadrupole moment is an excellent tool to investigate deformation and collective behavior in nuclei. It provides a direct link to the nuclear deformation-contrary to the electric quadrupole transition strength $B(E 2)$ - because it probes the charge distribution of a single nuclear state. Developing reliable methods to measure quadrupole moments of exotic nuclear states can therefore make a significant contribution to our insight into their structure.

The evolution of shell model orbits as a function of isospin induces shape changes and leads to new regions of deformation. An onset of deformation has been suggested for the neutron-rich $N \approx 40 \mathrm{Cr}$ and $\mathrm{Fe}(Z=24,26)$ isotopes, based on the lowering of the $2^{+}$excitation energies when approaching $N=40[1,2]$. Measuring quadrupole moments in this mass region will directly probe the nuclear deformation and serve as a sensitive test for nuclear models.
Previous studies of isomeric $g$ factors [3-5] have shown that the spin alignment, obtained from in-flight selected fragmentation beams, offers the opportunity to investigate nuclear moments of isomeric states. This paper presents the first measurement of the quadrupole moment of an isomer, populated in a fragmentation reaction, by application of the time dependent perturbed angular distribution (TDPAD) technique. Although the method is well known [6,7], its application to fragment beams selected with a nonzero degree separator requires a dedicated setup for each individual isomeric state. As a first test case, the $I^{\pi}=9 / 2^{+}$isomer in ${ }^{61} \mathrm{Fe}$ is investigated. It has a dominant $\nu g_{9 / 2}$ configuration, which was confirmed by a recent $g$ factor measurement [5]. Comparison of the measured $g$ factor with large scale shell model calculations in a fpg model space suggested a large spectroscopic quadrupole moment and thus a deformed structure for this intruder isomeric state [5].

The ${ }^{61} \mathrm{Fe}$ isomers were populated and aligned following the fragmentation of a $64.6 \mathrm{MeV} /$ nucleon ${ }^{64} \mathrm{Ni}$ beam (mean intensity $1.3 \mu \mathrm{A}$ ) impinging on a ${ }^{9} \mathrm{Be}$ target of $94.1 \mathrm{mg} / \mathrm{cm}^{2}$. In order to get the highest possible alignment, the fully stripped fragments were selected by the LISE [8] fragment separator in the outermost wing of the longitudinal momentum distribution. The experimental setup (Fig. 1), designed especially for this quadrupole moment measurement, was placed at the first focal plane of the separator. A $50 \mu \mathrm{m}$ thin plastic scintillator was used to monitor the implantation rate $\left(\approx 1.5 \times 10^{4} \mathrm{pps}\right)$ into the $\mathrm{Cd}$ host and to determine the ion arrival time. The $\gamma$-rays 


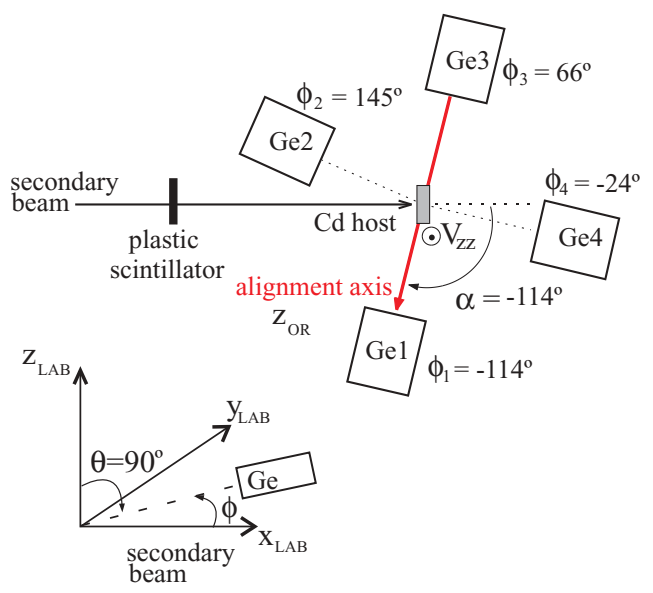

FIG. 1. (Color online) Schematic drawing of the experimental setup and definition of the laboratory (LAB) reference frame. See the text for the explanation on the choice of the detector angles.

deexciting the isomer were recorded with four single crystal germanium detectors, positioned in the horizontal plane at about $8 \mathrm{~cm}$ from the Cd host. Time and energy spectra were collected on an event-by-event basis during $36 \mathrm{~h}$, with the trigger for a valid event given by an ion- $\gamma$ coincidence within a $3 \mu$ s time window.

The $9 / 2^{+}$isomer in ${ }^{61} \mathrm{Fe}$ deexcites via a cascade of a $654 \mathrm{keV} M 2$ transition and a $207 \mathrm{keV} M 1$ transition (Fig. 2). Time spectra $I_{i}(t)(i=1 \rightarrow 4)$, gated by these two transitions, were constructed and used to generate the ratio functions $R(t)$ (Fig. 2):

$$
R(t)=\frac{I_{13}(t)-\epsilon I_{24}(t)}{I_{13}(t)+\epsilon I_{24}(t)},
$$

with $I_{i j}(t)=I_{i}(t)+I_{j}(t)$ representing the sum of the time spectra of detectors $i$ and $j$. The time spectra were normalized by a scaling factor $\varepsilon$ to correct for the different efficiencies of the detectors.

The $\gamma$-ray intensity of the transitions depopulating the isomeric state is given by $I_{i}(t)=I_{0} e^{-t / \tau} W\left(\theta_{i}, \phi_{i}, t\right)$, with $I_{0}$ the intensity at $t=0, \tau$ the lifetime of the isomeric state and $\left(\theta_{i}, \phi_{i}\right)$ the spherical coordinates of detector $i$ with respect to the laboratory (LAB) reference frame as defined in
Fig. 1. The detectors are positioned in the horizontal plane, thus $\theta_{i}=90^{\circ}$,

$$
W\left(\theta_{i}, \phi_{i}, t\right)=\sum_{\substack{k, n \\ k=\text { even }}} \sqrt{\frac{4 \pi}{2 k+1}} A_{k} U_{k} B_{k}^{n}(t) Y_{k}^{n}\left(\theta_{i}, \phi_{i}\right)
$$

represents the perturbed angular distribution [9] and completely defines the $R(t)$ function, since $I_{0} e^{-t / \tau}$ cancels out when taking the ratio in expression (1). $A_{k}$ are the angular distribution coefficients which depend on the multipolarity of the observed $\gamma$-transition as well as on the initial and final spins. The deorientation coefficients $U_{k}$ quantify the orientation loss due to preceding transitions. All the information on the perturbing interaction is contained in the orientation tensors $B_{k}^{n}(t)$, which describe the perturbed orientation of the nuclear ensemble in the isomeric state. They can be calculated as a function of the orientation at $t=0$ (time of implantation) [9]:

$$
B_{k}^{n}(t)=\sum_{\substack{k^{\prime}, n^{\prime} \\ k^{\prime}=\text { even }}} \mathrm{G}_{k k^{\prime}}^{n n^{\prime}}(t) \underbrace{\mathcal{D}_{n^{\prime} 0}^{k^{\prime}}\left(\alpha, 90^{\circ}, 0^{\circ}\right) B_{k^{\prime}}^{0}(t=0)_{O R}}_{B_{k^{\prime}}^{n^{\prime}}(t=0)} .
$$

$\mathrm{G}_{k k^{\prime}}^{n n^{\prime}}(t)$ are the perturbation coefficients and their specific form is determined by the quadrupole interaction. The aligned nuclear ensemble, produced in a fragmentation reaction, is axially symmetric and completely characterized by the orientation tensors $B_{k^{\prime}}^{0}(t=0)_{O R}$ with $k^{\prime}$ even. The index OR (orientation) indicates that these tensors are described in a reference frame with the alignment axis (i.e., the symmetry axis of the aligned ensemble) as the $\mathrm{Z}$-axis. The orientation tensors in the LAB frame $B_{k^{\prime}}^{n^{\prime}}(t=0)$ can be obtained by the transformation $\mathcal{D}_{n^{\prime} 0}^{k^{\prime}}\left(\alpha, 90^{\circ}, 0^{\circ}\right)$, with $\alpha$ the rotation angle of the alignment axis due to the Larmor precession in the separator dipole magnets (see Fig. 1 and [4]). The rotation matrix $\mathcal{D}_{n^{\prime} 0}^{k^{\prime}}\left(\alpha, 90^{\circ}, 0^{\circ}\right)$ is defined according to the convention of [10]. The amplitude of a quadrupole perturbed $R(t)$ function depends on the position of the detectors with respect to the alignment axis: combining detectors placed parallel and perpendicular to the alignment axis leads to the highest amplitude of the $R(t)$ function. The $g$ factor dependence of $\alpha$ [4] therefore makes the optimal experimental geometry for a quadrupole moment measurement case dependent. Using the known $g$ factor of the ${ }^{61} \mathrm{Fe}\left(9 / 2^{+}\right)$isomer [5], we find $\alpha=-114^{\circ}$ (Fig. 1). The detectors were placed accordingly, (a)

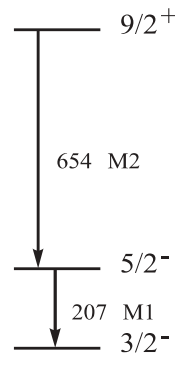

${ }^{61 \mathrm{Fe}}$
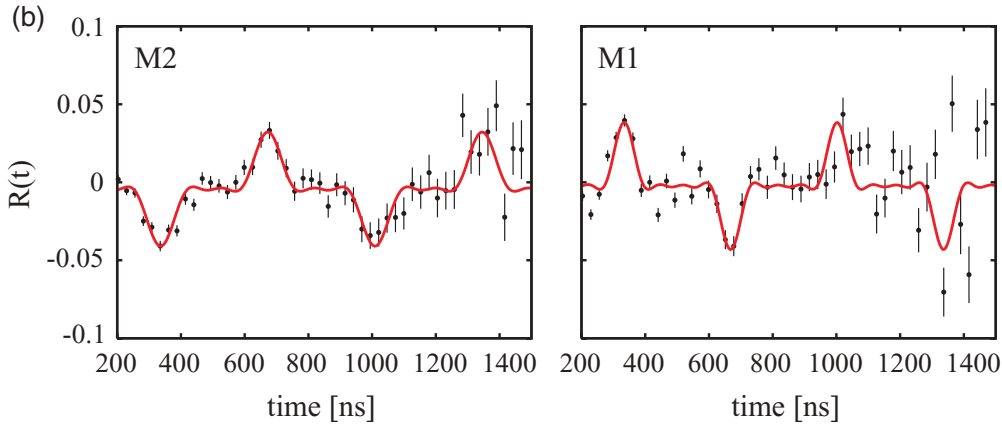

FIG. 2. (Color online) (a) Decay scheme of the ${ }^{61} \mathrm{Fe}\left(9 / 2^{+}\right)$isomer [5] (b) $R(t)$ functions for the $M 2\left(9 / 2^{+} \rightarrow 5 / 2^{-}\right)$and $M 1\left(5 / 2^{-} \rightarrow 3 / 2^{-}\right)$ transitions deexciting the $9 / 2^{+}$isomer. The axially symmetric electric field gradient of the Cd host was oriented perpendicular to the detector plane. 
with the exception of detector 2, which could not be placed that close to the beam line. However, the misalignment of $11^{\circ}$ is comparable to the angular coverage of a detector and only caused a 5\% reduction in the amplitude and no change in the shape of the $R(t)$ function.

For the interaction between the spectroscopic nuclear quadrupole moment $Q_{s}$ and an axially symmetric electric field gradient, the perturbation coefficients $\mathrm{G}_{k k^{\prime}}^{n n^{\prime}}(t)$ reduce to a superposition of several harmonics with basic frequency $\omega_{0}=\frac{n 2 \pi v_{Q}}{4 I(2 I-1)}$ [9], where $I$ is the spin and $n=3(6)$ for integer (half-integer) spin. The period of the $R(t)$ function depends therefore on both the spin $I$ and the quadrupole interaction frequency $v_{Q}=e Q_{s} V_{z z} / h$, with $V_{z z}$ the electric field gradient (EFG) induced by the implantation host on the nuclei of interest. The spin of the isomer fixes the maximum number of contributing harmonics, while their relative amplitudes depend on the orientation of $V_{z z}$. The shape of the $R(t)$ function can thus be optimized by selecting the best orientation of $V_{z z}$. Previous studies [6] have demonstrated that $\mathrm{Cd}$ is a good implantation host for $\mathrm{Fe}$ isotopes with a $V_{z z}$ that allows us to observe at least one $R(t)$ period. Based on simulations, it was established that one full $R(t)$ period has the most distinctive and thus the best measurable shape for $V_{z z}$ oriented perpendicular to the detector plane.

The $R(t)$ functions are fitted by the theoretical expression calculated from the perturbed angular distributions of the detectors. A $\chi^{2}$-minimization procedure is used to determine the parameters and their statistical errors. The transitions deexciting the isomer have pure $M 2$ and $M 1$ multipolarities [5] and the $A_{k}$ and $U_{k}$ coefficients are calculated based on that assumption. For the $207 \mathrm{keV} M 1$ transition there is only an $A_{2}$ coefficient, while for the $654 \mathrm{keV} \mathrm{M2} \mathrm{transition} \mathrm{the} A_{4}$ term contributes as well. The term proportional to $A_{4} B_{2}$ in the angular distribution for the $M 2$ transition leads to a broadening of the peaks as compared to those for the $M 1$ transition (Fig. 2). Only the second order orientation tensor $B_{2}^{0}(t=0)_{O R}$ is taken into account in the fit, since the influence of the higher orders was found to be negligible. The only free parameters in the fit are therefore $B_{2}^{0}(t=0)_{O R}$ and $v_{Q}$. It should be pointed out that, for an aligned nuclear ensemble, it is not possible to measure the sign of $v_{Q}$ and therefore only the absolute value of $Q_{s}$ can be determined. The frequencies obtained from both fits are consistent within their error bars. In order to exclude any influence of the intermediate $5 / 2^{+}$level, the result from the $M 2$ transition is adopted as the final value: $\left|v_{Q}\left({ }^{61} \mathrm{Fe}, 9 / 2^{+}\right)\right|=$ 35.7(3) MHz. The amount of alignment, deduced from the fitted $B_{2}^{0}(t=0)_{O R}$, is $-10.5(9) \%$, normalized according to the convention from [11]. This is about $64 \%$ of the alignment observed in a $g$ factor measurement, performed during the same run, using a $\mathrm{Cu}$ host and in agreement with similar observations for ${ }^{54} \mathrm{Fe}^{m}$ [6].

The quadrupole moment of the ${ }^{61} \mathrm{Fe}\left(9 / 2^{+}\right)$isomer can be derived relative to the quadrupole moment of the ${ }^{57} \mathrm{Fe}\left(3 / 2^{-}\right.$, $98 \mathrm{~ns})$ isomer by comparing their quadrupole frequencies $\left(v_{Q}\left({ }^{57} \mathrm{Fe}\right.\right.$ in $\left.\left.\mathrm{Cd}, 3 / 2^{-}\right)=13.0(6) \mathrm{MHz}[12]\right)$ :

$$
\left|\frac{Q_{s}\left({ }^{61} \mathrm{Fe}, 9 / 2^{+}\right)}{Q_{s}\left({ }^{57} \mathrm{Fe}, 3 / 2^{-}\right)}\right|=\left|\frac{v_{Q}\left({ }^{61} \mathrm{Fe}, 9 / 2^{+}\right)}{v_{Q}\left({ }^{57} \mathrm{Fe}, 3 / 2^{-}\right)}\right|=2.75(13) .
$$

Comparison of electric field gradients, deduced from quadrupole frequency measurements on ${ }^{57} \mathrm{Fe}^{m}$ in different Fe compounds, with ab initio calculations for these EFG's, revealed that the earlier adopted quadrupole moment of ${ }^{57} \mathrm{Fe}^{m}$ was wrong [13]. This was recently confirmed by a combination of atomic and nuclear calculations [14], which conclude that the quadrupole moment for the ${ }^{57} \mathrm{Fe}$ isomer is $Q_{s}=+15(2) e \mathrm{fm}^{2}$. With this value, we obtain $\mid Q_{s}\left({ }^{61} \mathrm{Fe}\right.$, $\left.9 / 2^{+}\right) \mid=41(6) e \mathrm{fm}^{2}$. Although the quadrupole frequency could be measured with a precision better than $1 \%$, it is the poor knowledge of the reference quadrupole moment which defines the final result.

Large scale shell model calculations (LSSM) for ${ }^{61} \mathrm{Fe}$ were performed by Matea et al. [5]. The first $9 / 2^{+}$state was obtained at $720 \mathrm{keV}$ with a spectroscopic quadrupole moment $Q_{s}=-58 e \mathrm{fm}^{2}$, slightly overestimating the deformation. The same interaction and effective charges $\left(e_{\pi}=1.5 e\right.$ and $\left.e_{v}=0.5 e\right)$ were used successfully $[15,16]$ to reproduce the $B(E 2)$ transition strengths of neutron-rich Ni isotopes up to $N=40$. A systematic comparison between measured and calculated quadrupole moments in this region would aid in checking the validity of these effective charges and test the predictive power of the considered effective interaction.

To gain more insight into the intrinsic deformation at low energies in ${ }^{61} \mathrm{Fe}$, mean-field based calculations have been performed using a new parametrization [17] of the Gogny force [18]. Hartree-Fock-Bogoliubov (HFB) axially symmetric solutions have been obtained using the blocking procedure for all one-quasiparticle (qp) neutron states originating from the $f_{5 / 2}, p_{1 / 2}, p_{3 / 2}$, and $g_{9 / 2}$ orbits. The axial HFB calculations were extended to investigate the stiffness of the $K^{\pi}$ solutions against axial deformation and it was found that this property very much depends upon the blocked configurations. Configuration mixing calculations were performed separately for each blocked configuration using the Generator Coordinate Method (GCM) implemented with the Gaussian Overlap Approximation (GOA) [19]. The predicted excitation energies and intrinsic charge quadrupole moments $Q_{0}$ of the obtained $K^{\pi}$ levels are shown in Fig. 3. It should be noted that no effective charge is assumed in these calculations. The predictions cover a wide range of deformations from oblate $\left(K^{\pi}=9 / 2^{+}\right)$to prolate $\left(K^{\pi}=1 / 2^{+}\right)$ shapes. A good agreement is found with the experimental spin-parity/assignments $[5,20]$ of the lowest negative-parity states. The $K^{\pi}=3 / 2^{-}$ground state is predicted to have prolate deformation with $Q_{0}=51 e \mathrm{fm}^{2}$, corresponding to a weak deformation $\beta_{2}=+0.11$. The positive parity states originating from the neutron $g_{9 / 2}$ orbital are predicted with either prolate $(1 / 2 \leqslant K \leqslant 5 / 2)$ or oblate $(7 / 2 \leqslant K \leqslant 9 / 2)$ shapes, leading to two possible deformations for the observed $9 / 2^{+}$isomeric state. In the strong coupling limit, the spectroscopic quadrupole moment for an $I^{\pi}=9 / 2^{+}$state built on the $K^{\pi}=1 / 2^{+}, 3 / 2^{+}$, or $9 / 2^{+}$level is $Q_{s}=-36,-30$, or $-46 e \mathrm{fm}^{2}$, respectively. All absolute values of these spectroscopic quadrupole moments are consistent with the measured value, $\left|Q_{s}\right|=41(6) e \mathrm{fm}^{2}$, thus confirming that the $9 / 2^{+}$isomer is moderately deformed with $\beta_{2} \approx-0.21$ or $\beta_{2} \approx+0.24$. The weak deformation predicted for the ground state is at variance with the conclusions based on $2^{+}$excitation 


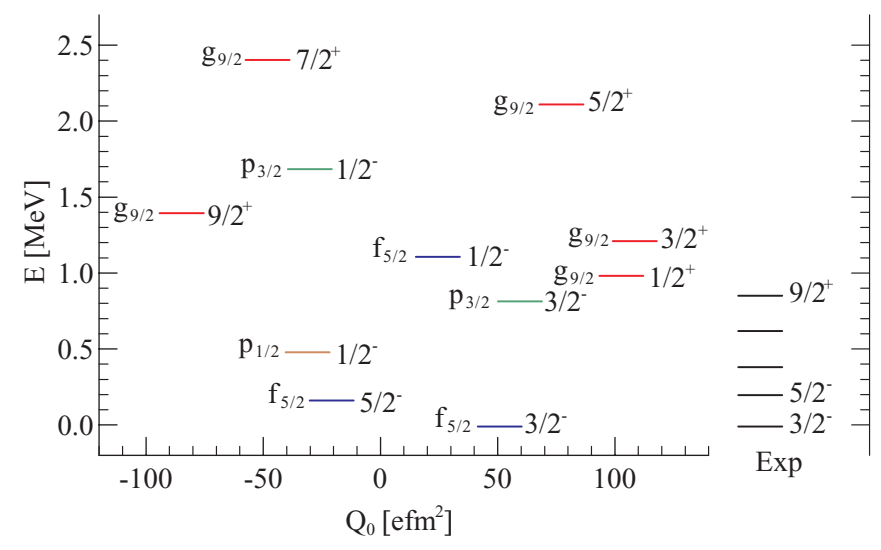

FIG. 3. (Color online) Excitation energies and intrinsic charge quadrupole moments from GCM + GOA calculations. Onequasiparticle excitations, labeled by $K^{\pi}$ and their related spherical orbital, are compared to the experimental level scheme (data taken from $[5,20])$.

energies and $B(E 2)$ values in this mass region, which suggest a deformation close to $\beta_{2} \approx 0.2$ [21]. The measurement of the ground state quadrupole moment would allow a direct comparison of the isomeric and ground state deformation and could eventually reveal the coexistence of different shapes at low energy in ${ }^{61} \mathrm{Fe}$.

More insight into the oblate or prolate nature of the ${ }^{61} \mathrm{Fe}$ isomer can be gained by studying the band built on top of the $9 / 2^{+}$isomer. Such studies were performed already in the ${ }^{57,59} \mathrm{Fe}$ isotopes [22,23], favoring a prolate decoupled band on top of the $9 / 2^{+}$state. On the other hand, Deacon et al. [24] proposed a prolate to oblate shape transition to appear at $N=35$ for the $9 / 2^{+}$states in the adjacent $\mathrm{Cr}$ isotopes. The availability of such measurements for ${ }^{61} \mathrm{Fe}$ would help to reach a deeper understanding of this suggested shape transition.

In conclusion, it was possible for the first time to measure the static quadrupole moment of a neutron-rich isomeric state produced and spin-aligned in an intermediate energy projectile fragmentation reaction. The extracted value of $\left|Q_{S}\right|$ provides experimental proof for the moderate deformation of the ${ }^{61} \mathrm{Fe}^{m}$ isomer and demonstrates the onset of deformation when the $v g_{9 / 2}$ intruder orbital comes into play. This experiment illustrates the applicability of the method to microsecond isomers $\left(T_{1 / 2}=200 \mathrm{~ns}-10 \mu \mathrm{s}\right)$, produced at rates of a few thousands per second. In combination with reliable $a b$ initio calculations of electric field gradients, which are nowadays available, this opens the way for investigations on more exotic cases.

We thank the technical staff of the accelerator facility at GANIL for their help and the IN2P3/EPSRC French/U.K. loan pool for providing the Ge detectors. We are grateful to J. A. Ropert and G. Voltolini for their assistance with the filling system for the Ge detectors and to D. Grebille for checking the quality of the $\mathrm{Cd}$ single crystal. This work was partially supported by the FP6-EURONS project no. RII3-CT-2004506065 , the IUAP project P5/07 of OSCT Belgium, and the FWO-Vlaanderen. The Weizmann group has been supported by the Israel Science foundation. D.L.B. is partly supported by the Bulgarian Science Fund under contract VUF06/05.
[1] M. Hannawald et al., Phys. Rev. Lett. 82, 1391 (1999).

[2] O. Sorlin et al., Eur. Phys. J. A 16, 55 (2003).

[3] W.-D. Schmidt-Ott et al., Z. Phys. A 350, 215 (1994).

[4] G. Georgiev et al., J. Phys. G 28, 2993 (2002).

[5] I. Matea et al., Phys. Rev. Lett. 93, 142503 (2004).

[6] M. Hass et al., Nucl. Phys. A414, 316 (1984).

[7] G. Goldring and M. Hass, in Treaties on Heavy-Ion Science, Vol. 3, 539, edited by D. Allan Bromley (Plenum Press, New York, 1985).

[8] R. Anne et al., Nucl. Instrum. Methods Phys. Res. A 257, 215 (1987).

[9] R. M. Steffen and K. Alder, in The Electromagnetic Interaction in Nuclear Spectroscopy, edited by W. D. Hamilton (North Holland, Amsterdam, 1975), Chaps. 12 and 13.

[10] D. M. Brink and G. R. Satchler, Angular Momentum (Oxford University Press, Oxford, 1968).

[11] G. Neyens, Rep. Prog. Phys. 66, 633 (2003).

[12] R. Vianden, Hyperfine Interact. 35, 1079 (1987).
[13] P. Dufek, P. Blaha, and K. Schwarz, Phys. Rev. Lett. 75, 3545 (1995).

[14] G. Martinez-Pinedo et al., Phys. Rev. Lett. 87, 062701 (2001).

[15] O. Sorlin et al., Phys. Rev. Lett. 88, 092501 (2002).

[16] E. Caurier et al., Eur. Phys. J. A 15, 145 (2002).

[17] F. Chappert and M. Girod, Int. J. Mod. Phys. E 15, 339 (2006).

[18] J.-F. Berger, M. Girod, and D. Gogny, Comput. Phys. Commun. 63, 365 (1991); J. Dechargé and D. Gogny, Phys. Rev. C 21, 1568 (1980).

[19] J. Libert, M. Girod, and J. P. Delaroche, Phys. Rev. C 60, 054301 (1999).

[20] M. R. Bhat, Nucl. Data Sheets 88, 417 (1999).

[21] S. Raman et al., At. Data Nucl. Data Tables 78, 1 (2001).

[22] A. M. Nathan, J. W. Olness, E. K. Warburton, and J. B. McGrory, Phys. Rev. C 17, 1008 (1978).

[23] E. K. Warburton, J. W. Olness, A. M. Nathan, J. J. Kolata, and J. B. McGrory, Phys. Rev. C 16, 1027 (1977).

[24] A. N. Deacon et al., Phys. Lett. B622, 151 (2005). 